\title{
Análise bayesiana para modelos de degradabilidade ruminal
}

\author{
Bayesian analisys for ruminal degradability models
}

\author{
Taciana Villela Savian $^{{ }^{*}}$ Joel Augusto Muniz ${ }^{\mathrm{I}}$ Thelma Sáfadi ${ }^{\mathrm{I}}$ Fabyano Fonseca e Silva ${ }^{\mathrm{II}}$
}

RESUMO

Neste estudo, utilizou-se a metodologia bayesiana para ajustar os modelos de ORSKOV \& MCDONALD (1979) $e$ MCDONALD (1981) a conjuntos de dados simulados e a um conjunto de dados de porcentagem de degradação da fibra em detergente neutro da gramínea coastcross (Cynodon dactylon $x$ Cynodon nlemfuensis), ao longo do tempo. As amostras das distribuições marginais a posteriori dos parâmetros foram obtidas por meio dos métodos de Monte Carlo com cadeias de Markov (MCMC), especificamente, os algoritmos Amostrador de Gibbs e Metropolis-Hastings. A metodologia bayesiana mostrou-se eficiente, sendo avaliada $e$ comprovada pelo estudo de simulação, que apresentou estimativas bem próximas ao valor paramétrico. As estimativas obtidas para os parâmetros dos modelos por meio da abordagem bayesiana mostraram-se bastante coerentes com os valores relatados na literatura. O modelo de Orskov e McDonald foi mais plausível que o modelo de McDonald na descrição dos dados de degradação.

Palavras-chave: modelo não linear, degradabilidade in situ, métodos MCMC, inferência bayesiana.

\section{ABSTRACT}

The bayesian methodology was used to estimate the parameters of ORSKOV \& MCDONALD (1979) and MCDONALD (1981) models. A study was conducted by using both simulated and real data percentage of coastcross grass (Cynodon dactylon $x$ Cynodon nlemfuensis) fiber degradation with neutral detergent fiber degradation over the time. The posterior marginal samples distributions for the parameters were obtained by Gibbs Sampler and Metropolis-Hastings algorithms. The bayesian approach, evaluated and verified by the simulation studied, has proved to be efficient and the parameter estimated were quite close to the parametric values. The parameters estimated for both models using bayesian approach from real data were fairly consistent with the values reported in the literature. The Orskov and McDonald model was more plausible than the description degradation data made by the McDonald model.

Key words: nonlinear model, in situ degradability, MCMC methods, bayesian inference.

\section{INTRODUÇÃO}

A caracterização do alimento de acordo com sua composição química e constituição de suas diferentes frações degradáveis ou não no rúmen é o grande objetivo dos nutricionistas para alcançar com êxito o balanceamento de rações que proporcionem nutrientes para o crescimento e desenvolvimento dos microrganismos do rúmen e para o animal. Portanto, técnicas in vivo, in vitro e in situ têm sido utlizadas para determinar o aproveitamento das diferentes frações dos alimentos.

A técnica in situ consiste em determinar o desaparecimento de componentes da amostra de alimentos acondicionados em sacos de náilon e incubados no rúmen por períodos variáveis de tempo. Segundo MERTENS (1993), as primeiras avaliações de processos de digestão, considerando-se os tempos de retenção, eram qualitativas e baseavam-se na interpretação visual de curvas de digestão, sendo de difícil descrição, pois essas curvas mostravam comportamentos não lineares.

'Departamento de Ciências Exatas, Universidade Federal de Lavras (UFLA), 37200-000, Lavras, MG, Brasil. E-mail: tacianavillela@gmail.com.*Autor para correspondência.

"Departamento de Informática, Universidade Federal de Viçosa (UFV), Viçosa, MG, Brasil. 
ORSKOV \& MCDONALD (1979) sugerem um modelo exponencial baseado no princípio de redução do material durante a incubação no estudo de degradação, em função do tempo de incubação no rúmen, dado por: $\operatorname{Deg}_{i}=a+b\left(1-e^{-c t_{i}}\right)$, em que: $\operatorname{Deg}_{i}$ é a degradação do alimento até o tempo $i$; $t$ é o tempo de incubação das amostras, com $i=1, \ldots, n ; n$ é o número de coleta das amostras; $a$ é a fração solúvel do componente nutritivo analisado; $b$ é a fração potencialmente degradável do componente nutritivo analisado; $c$ é a taxa de degradação da fração $b$ e $\varepsilon_{i}$ é erro aleatório com distribuição normal de média zero e variância $\sigma^{2}$.

Determinadas frações de alguns alimentos apresentam um período de latência (L), ou lag time, em que não se verifica a degradação do substrato. Durante esse período, pode ocorrer hidratação das partículas do alimento, remoção de substâncias inibidoras, eventos ligados à adesão e efetiva colonização das partículas do alimento pelos microrganismos ruminais. Desse modo, é justificável a introdução de novos modelos que determinem a contribuição do período de latência $(L)$ sobre a cinética de degradação ruminal. Com base nisso, MCDONALD (1981) revisou o modelo e propôs a inclusão do tempo de colonização, dado por: $\operatorname{Deg}_{i}=a+b\left(1-e^{-c\left(t_{i}-L\right)}\right)+\varepsilon_{i}$, para $t_{i}>L$, em que: $\operatorname{Deg}_{i}$, $a, b, c, t_{i}$ e $\varepsilon_{i}$ foram definidos anteriormente e $L$ é o lag time.

As estimativas dos parâmetros dos modelos são utilizadas para calcular as degradabilidades potencial $(D P)$ e efetiva $(D E)$, dadas, respectivamente,

por: $D P=a+b$ e $D E=a+\frac{b c}{c+k}$, em que: $k$ é a taxa de

passagem. A DP representa a quantidade de alimento que pode se solubilizar ou degradar dentro do rúmen se o tempo não for um fator limitante.

ORSKOV (1982) cita que, em condições normais, a digestão é resultante de duas forças competitivas que atuam simultaneamente, a taxa de passagem $(k)$ e a taxa de degradação da fração potencialmente degradável (c). A inclusão da taxa de passagem no cálculo da degradação origina a degradabilidade efetiva $(D E)$.

O ajuste de modelos não lineares na descrição da degradação ruminal foi investigado, sob o aspecto metodológico, por SAVIAN et al. (2007a, 2007b, 2007c), utilizando a abordagem clássica. Alguns trabalhos, na área zootécnica, que abordam a estimação de parâmetros em modelos não lineares pelo método bayesiano (PAIVAet al., 2005; MUNIZ et al., 2007; SILVA et al., 2008) demonstram grande sucesso pela possibilidade de ser possível modelar da incerteza de todas as quantidades desconhecidas no modelo, o que é feito por meio da distribuição a priori atribuída aos parâmetros.

A diminuição da dependência do ajuste do modelo em relação ao número de dados utilizados devese principalmente ao fato de que o conceito de graus de liberdade residuais não se aplica para tal abordagem (SILVA et al., 2005; SILVA et al., 2006; MARTINS FILHO et al., 2008), uma vez que o ajuste pode ser sustentado por distribuições a priori altamente informativas, as quais refletem diretamente a falta de informação proveniente dos dados amostrais.

PAULINO et al. (2003) consideram que, em algumas situações, o conhecimento a priori é pouco significativo em relação à informação amostral, e o pesquisador é conduzido a adotar distribuições a priori minimamente informativas, também chamadas de distribuições a priori não informativas. Com uma priori estabelecida para um parâmetro (ou vetor de parâmetros) $P(\theta)$ e a verossimilhança da amostra (dados), L $(\theta \mid \mathrm{Y})$, é possível, por meio do teorema de Bayes, atualizar a incerteza a priori, obtendose a distribuição a posteriori, a qual é dada por:

$P(\theta \mid Y)=\frac{L(\theta \mid Y) P(\theta)}{\int L(\theta \mid Y) P(\theta) d \theta}$, sendo $\mathrm{Y}=\left\{y_{1}, y_{2}, \ldots, y_{n}\right\} \mathrm{e}$ $\theta$ um vetor de parâmetros.

Para se inferir qualquer elemento de um vetor de parâmetros, é necessário obter a sua distribuição $a$ posteriori marginal. Para isso, a distribuição $a$ posteriori conjunta deve ser integrada em relação a todos os outros elementos do vetor paramétrico (ROSA, 1998). A integração dessa distribuição, para obtenção das distribuições marginais, pode ser muito complicada, sendo então necessário o uso de algoritmos especiais como, por exemplo, o Gibbs Sampler e Metropolis-Hastings, os quais fazem parte de uma classe de algoritmos denominada de MCMC (Markov Chain-Monte Carlo).

Esses algoritmos têm a habilidade de gerar cadeias que possuem distribuição estacionária. As cadeias resultantes necessitam ter sua convergência diagnosticada. Os métodos comumente utilizados para diagnosticar a convergência são aqueles propostos por: GELMAN \& RUBIN (1992), RAFTERY \& LEWIS (1992) e GEWEKE (1992). Uma avaliação detalhada dos critérios de convergência pode ser encontrada em NOGUEIRA et al. (2004). Análises gráficas também são utilizadas para diagnosticar a convergência.

A comparação dos modelos é comumente realizada pelo Fator de Bayes (FB). Em diversas situações, o pesquisador, interessado em avaliar a consistência dos modelos com os dados, fornece a 
mesma probabilidade a priori para cada modelo. Nesse caso, o Fator de Bayes é dado pela razão das verossimilhanças marginais. A resolução da integral envolvida na obtenção da verossimilhança marginal geralmente não é analítica, e uma possível solução é considerar os valores de $\theta$ gerados via algoritmos MCMC (KASS \& RAFTERY, 1995).

Em relação à interpretação do fator de Bayes, pode-se dizer que esta é simples e direta, pois, se $\mathrm{FB}_{(M i, M i}$ é maior que 1 , tem-se a indicação que o modelo disposto no numerador, $M_{i}$, é mais plausível ou verossímil que o modelo disposto no denominador, $M_{j}$. Caso contrário, o modelo no denominador é mais plausível que o modelo disposto no numerador. Uma interpretação para o fator de Bayes é dada por JEFFREYS (1961). Segundo o autor, o fator de Bayes contém categorias de evidências em relação aos modelos $M_{i}$ e $M_{j}$, podendo ser utilizado na escolha do melhor modelo.

Em razão da escassez de trabalhos na área de nutrição animal, especificamente em estudos de cinética de degradação ruminal que são tratados com a abordagem bayesiana, e do fato de se tratar de uma metodologia versátil, menos dependente das pressuposições usuais exigidas em métodos estatísticos clássicos, o presente trabalho objetivou estudar a cinética de degradação ruminal, por meio dos modelos não lineares de ORSKOV \& MCDONALD (1979) e MCDONALD (1981), e apresentar um procedimento bayesiano para a análise e comparação desses modelos.

\section{MATERIAL E MÉTODOS}

Para ilustrar a metodologia de ajuste, foram utilizados dados parciais de um experimento de degradabilidade conduzido por REIS (2000). Nesse experimento, foi utilizada a técnica de degradabilidade in situ, em que a porcentagem de degradação da FDN da gramínea coastcross, com idade de corte de 120 dias, foi avaliada em nove tempos de incubação $(0,3,6,12$, 24, 48, 72, 96 e 120 horas).

Considerando o modelo de ORSKOV \& MCDONALD (1979), foram simulados os valores de degradação da FDN em oito cenários diferentes. Os valores paramétricos foram: $a$ igual a $6 \%$ e $20 \%$; $b$ igual a $40 \%$ e $60 \%$, $c$ igual a $0,03 \% / h$ e $0,04 \% / h$ e $\varepsilon_{i} \sim N(0,2)$. Para o modelo de MCDONALD (1981), foram utilizados os mesmos valores paramétricos descritos anteriormente, e para o parâmetro L foi utilizado um valor fixo de três horas.

Para a obtenção da distribuição a posteriori conjunta dos parâmetros dos modelos de Orskov e McDonald e McDonald, assumiram-se prioris uniformes para os parâmetros $a, b$ e $c$. Para a precisão $\tau$, dada pelo inverso da variância $\sigma^{2}$, assumiu-se como priori uma distribuição Gama com hiperparâmetros $\alpha$ e $\beta$ iguais a 250 e 500, respectivamente. Para o parâmetro L, no modelo de McDonald, assumiu-se como priori uma função indicadora do tipo $\mathrm{I}_{(L>0)}$. As distribuições $a$ priori foram especificadas com base em resultados de estudos anteriores que utilizaram conjuntos de dados semelhantes e os mesmos modelos, pois esse processo de atualização de informação via análises prévias constitui uma importante característica da análise Bayesiana.

Os algoritmos para o amostrador de Gibbs e o Metropolis-Hastings foram implementados utilizando-se a linguagem R (R DEVELOPMENT CORE TEAM, 2007). O total de iterações consideradas, bem como o descarte (burn-in) e espaçamento entre pontos amostrados (thin), tanto para dados simulados, como para os dados reais, foram obtidos por meio do critério de RAFTERY \& LEWIS (1992) na análise de uma amostra piloto com 10.000 iterações. Para os valores estipulados, em cada situação, a condição de convergência das cadeias foi verificada por meio dos critérios de GEWEKE (1992), GELMAN \& RUBIN (1992) e RAFTERY \& LEWIS (1992), que estão disponíveis no pacote BOA (Bayesian Output Analysis) do software R.

Amostras das distribuições marginais $a$ posteriori para $D E$ e $D P$ foram obtidas pela substituição de $a, b$ e $c$ pelas suas respectivas estimativas, a cada iteração dos algoritmos, obtendo-se, assim, ao final do processo, um conjunto de valores representativos dessas distribuições.

A comparação dos modelos, no ajuste aos dados reais, foi feita pelo fator de Bayes (FB), utilizando os valores gerados pelos métodos MCMC para obter as estimativas da verossimilhança marginal, procedimento sugerido por KASS \& RAFTERY (1995). A interpretação do fator foi feita segundo sugerido por JEFFREYS (1961), em que: se $F B_{(M i, M j)}<1$ há evidência a favor de $M_{j}$; se $1 \leq F B_{(M i, M j)}<3,2$ há evidência muito fraca a favor de $M$; se 3,2 $\leq F B_{(M i, M j)}<10$ há evidência fraca a favor de $M_{i}^{i}$; se $10 \leq F B_{(M i, M j)}<100$ há evidência forte a favor de $M_{i}$; e se $F B_{(M i, M j)} \geq 100$ há evidência muito forte a favor de $M_{i}$.

\section{RESULTADOS E DISCUSSÃO}

A partir da distribuição a posteriori conjunta dos parâmetros do modelo de Orskov e McDonald, foram obtidas as distribuições condicionais completas a posteriori, nas quais foi aplicada a amostragem de Gibbs (parâmetros $a, b$ e $\tau$ ) e o algoritmo MetropolisHastings (parâmetro $c$ ) e são representadas por: 


$$
\begin{aligned}
& a \mid b, c, \tau, \alpha, \beta \sim N\left(\frac{\sum_{i=1}^{n}\left(y_{i}-b\left(1-\exp \left(-c t_{i}\right)\right)\right)}{n}, \frac{\sigma^{2}}{n}\right) \\
& b \mid a, c, \tau, \alpha, \beta \sim N\left(\frac{\sum_{i=1}^{n}\left(y_{i}-a\right)\left(\left(1-\exp \left(-c t_{i}\right)\right)\right)}{\sum_{i=1}^{n}\left(1-\exp \left(-c t_{i}\right)\right)^{2}}, \frac{\sigma^{2}}{\sum_{i=1}^{n}\left(1-\exp \left(-c t_{i}\right)\right)^{2}}\right) \\
& p(c \mid a, b, \tau, y, \alpha, \beta) \propto \exp \left[-\frac{\tau}{2} \sum_{i=1}^{n}\left\{y_{i}-a-b\left(1-\exp \left(-c t_{i}\right)\right)\right\}^{2}\right] \text { (3) } \\
& \tau \mid a, b, c, y, \alpha, \beta \sim G\left(\frac{n+2 \alpha}{2}, \beta+\frac{1}{2} \sum_{i=1}^{n}\left(y_{i}-a-b\left(1-\exp \left(-c t_{i}\right)\right)\right)^{2}\right) .(4)
\end{aligned}
$$

A distribuição auxiliar utilizada no algoritmo Metropolis-Hastings (parâmetro $c$ ) foi uma distribuição gama com parâmetros $\alpha$ e $\beta$ iguais a 1.000 e 25.000, para o valor paramétrico de $0,04 \% \mathrm{~h}^{-1}$, e iguais a 3.000 e 100.000 , para o valor paramétrico de $0,03 \% \mathrm{~h}^{-1}$. Considerando que a média da distribuição gama é dada pelo quociente $\alpha$ / $\beta$, os valores dos parâmetros foram escolhidos de forma a garantir que a média dessa distribuição estivesse próxima do valor paramétrico utilizado e que fosse aproximadamente simétrica.

Para o modelo de McDonald, as distribuições condicionais completas a posteriori para cada parâmetro são dadas por:

$$
\begin{aligned}
& a \mid b, c, L, \tau, \alpha, \beta \sim N\left(\frac{\sum_{i=1}^{n}\left(y_{i}-b\left(1-\exp \left(-c\left(t_{i}-L\right)\right)\right)\right)}{n}, \frac{\sigma^{2}}{n}\right) \\
& b \mid a, c, L, \tau, \alpha, \beta \sim N\left(\frac{\sum_{i=1}^{n}\left(y_{i}-a\right)\left(\left(1-\exp \left(-c\left(t_{i}-L\right)\right)\right)\right)}{\sum_{i=1}^{n}\left(1-\exp \left(-c\left(t_{i}-L\right)\right)\right)^{2}}, \frac{\sigma^{2}}{\sum_{i=1}^{n}\left(1-\exp \left(-c\left(t_{i}-L\right)\right)\right)^{2}}\right)
\end{aligned}
$$

$p(c \mid a, b, L, \tau, y, \alpha, \beta) \propto$

$\exp \left[-\frac{\tau}{2} \sum_{i=1}^{n}\left\{y_{i}-a-b\left(1-\exp \left(-c\left(t_{i}-L\right)\right)\right)\right\}^{2}\right]$

$p(L \mid a, b, c, \tau, y, \alpha, \beta) \propto$

$\exp \left[-\frac{\tau}{2} \sum_{i=1}^{n}\left\{y_{i}-a-b\left(1-\exp \left(-c\left(t_{i}-L\right)\right)\right)\right\}^{2}\right] I_{(L>0)}$

$\tau \mid a, b, c, L, y, \alpha, \beta \sim$

$G\left(\frac{n+2 \alpha}{2}, \beta+\frac{1}{2} \sum_{i=1}^{n}\left(y_{i}-a-b\left(1-\exp \left(-c\left(t_{i}-L\right)\right)\right)\right)^{2}\right)$

Para o parâmetro $c$, a distribuição auxiliar, utilizada no algoritmo de Metropolis-Hastings, foi uma distribuição gama com parâmetros $\alpha$ e $\beta$ iguais a 2.000 e 50.000 no ajuste dos dados reais, e para o estudo de simulação os valores dos parâmetros $\alpha$ e $\beta$ foram os mesmos descritos no ajuste do modelo de ORSKOV \& MCDONALD(1979).
Para o parâmetro L, a distribuição auxiliar utilizada no algoritmo Metropolis-Hastings, tanto no ajuste aos dados reais, como no estudo de simulação, foi uma distribuição uniforme entre os limites 0 e 8 . Os valores dos parâmetros foram escolhidos de forma que o limite inferior garantisse que o valor do parâmetro fosse positivo e que o limite superior mantivesse uma interpretação coerente do parâmetro, uma vez que este é medido em horas.

Na tabela 1, podem ser visualizados os resultados do estudo de simulação a partir dos oito cenários propostos para o modelo de ORSKOV \& MCDONALD (1979). Para este estudo de simulação, apenas foram apresentados, como resultados do critério de convergência, aqueles propostos por GELMAN \& RUBIN (1992). Para avaliação desse critério, foram geradas, para cada parâmetro, duas cadeias a partir de valores iniciais distintos, e os valores estimados do fator de redução de escala $(R)$, em todos os cenários simulados, estiveram próximos de 1 e não apresentam evidência contra a convergência das cadeias.

Ainda na tabela 1 , observa-se que, sob os oito cenários simulados, as estimativas da média e moda a posteriori, além de estarem bastante próximas entre si, evidenciando distribuições do tipo simétricas, estão bastante próximas do valor verdadeiro dos parâmetros, demonstrando que a metodologia utilizada por ser adaptada às diferentes situações comumente encontradas em estudos de degradação ruminal.

Os resultados do estudo de simulação, a partir dos cenários considerados, para o modelo de MCDONALD (1981), estão apresentados na tabela 2. Os valores estimados de $R$, para o critério de Gelman e Rubin, em todos os cenários simulados, também foram próximos do valor 1, não apresentando evidência contra a convergência das cadeias. Os valores estimados da média e moda a posteriori, para os parâmetros $a$ e $b$ do modelo, apesar de estarem próximos entre si, evidenciando distribuições do tipo simétricas, estão menos próximos do valor verdadeiro dos parâmetros. Essa menor proximidade das estimativas em relação aos valores paramétricos pode ser explicada pela complexidade do modelo considerado. Para o parâmetro L (tempo de colonização), os valores estimados da média e moda a posteriori estão mais distantes entre si, retratando para esse parâmetro distribuições do tipo assimétricas.

Com relação ao ajuste dos modelos aos dados reais (Tabela 3), o critério proposto por GEWEKE (1992), que consiste em indicar a convergência da média a posteriori, apresentou cálculo do valor $p$ sempre maior que o nível de significância pré-fixado de 5\%, não apresentando evidências contra a convergência 
Tabela 1 - Critério de Gelman e Rubin ( $\hat{R}$ ), estimativas da média e moda a posteriori, intervalo de máxima densidade a posteriori (HPD) e Erro de Monte Carlo (EMC) para os dados simulados segundo o modelo de ORSKOV \& MCDONALD (1979).

\begin{tabular}{|c|c|c|c|c|c|c|}
\hline & \multicolumn{4}{|c|}{ 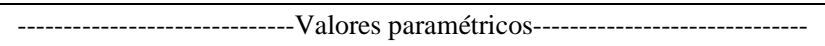 } & \multirow{2}{*}{ DP (\%) } & \multirow{2}{*}{ DE (\%) } \\
\hline & $a(\%)$ & $b(\%)$ & $C(\% / \mathrm{h})$ & $\tau$ & & \\
\hline & 6,0 & 40,0 & 0,03 & 0,5 & 46,0 & 21,0 \\
\hline$\hat{R}$ & 1,003 & 1,001 & 1,005 & 1,000 & 1,001 & 1,001 \\
\hline Média & 5,9 & 39,9 & 0,03 & 0,51 & 45,9 & 21,0 \\
\hline Moda & 5,9 & 40,0 & 0,03 & 0,51 & 45,9 & 21,0 \\
\hline HPD 95\% & {$[5,0 ; 6,8]$} & {$[39,0 ; 41,8]$} & {$[0,029 ; 0,031]$} & {$[0,45 ; 0,56]$} & {$[45,4 ; 46,4]$} & {$[20,6 ; 21,5]$} \\
\hline \multirow[t]{2}{*}{ EMC } & 0,01 & 0,01 & 0,00001 & 0,0005 & 0,005 & 0,005 \\
\hline & 6,0 & 40,0 & 0,04 & 0,5 & 46,0 & 23,8 \\
\hline$\hat{R}$ & 1,008 & 1,003 & 1,009 & 1,000 & 1,004 & 1,002 \\
\hline Média & 5,7 & 40,1 & 0,04 & 0,51 & 45,9 & 23,8 \\
\hline Moda & 5,7 & 40,1 & 0,04 & 0,50 & 45,9 & 23,8 \\
\hline HPD 95\% & {$[4,6 ; 6,8]$} & {$[39,0 ; 41,2]$} & {$[0,039 ; 0,042]$} & {$[0,45 ; 0,56]$} & {$[45,5 ; 46,3]$} & {$[23,3 ; 24,2]$} \\
\hline \multirow[t]{2}{*}{ EMC } & 0,02 & 0,02 & 0,00003 & 0,0005 & 0,004 & 0,006 \\
\hline & 6,0 & 60,0 & 0,03 & 0,5 & 66,0 & 28,5 \\
\hline$\hat{R}$ & 1,003 & 1,004 & 1,001 & 1,000 & 1,000 & 1,000 \\
\hline Média & 5,9 & 59,9 & 0,03 & 0,51 & 65,8 & 28,5 \\
\hline Moda & 5,9 & 59,9 & 0,03 & 0,50 & 65,8 & 28,5 \\
\hline HPD 95\% & {$[4,9 ; 6,7]$} & {$[59,0 ; 61,0]$} & {$[0,029 ; 0,031]$} & {$[0,45 ; 0,56]$} & {$[65,3 ; 66,4]$} & {$[28,1 ; 28,9]$} \\
\hline \multirow[t]{2}{*}{ EMC } & 0,01 & 0,01 & 0,00001 & 0,0005 & 0,005 & 0,005 \\
\hline & 6,0 & 60,0 & 0,04 & 0,5 & 66,0 & 32,7 \\
\hline$\hat{R}$ & 1,004 & 1,003 & 1,003 & 1,000 & 1,001 & 1,000 \\
\hline Média & 5,6 & 60,2 & 0,04 & 0,51 & 65,8 & 32,7 \\
\hline Moda & 5,7 & 60,2 & 0,04 & 0,50 & 65,8 & 32,7 \\
\hline HPD 95\% & {$[4,5 ; 6,8]$} & {$[59,1 ; 61,3]$} & {$[0,039 ; 0,042]$} & {$[0,45 ; 0,57]$} & {$[65,4 ; 66,3]$} & {$[32,2 ; 33,1]$} \\
\hline \multirow[t]{2}{*}{ EMC } & 0,01 & 0,01 & 0,00002 & 0,0005 & 0,004 & 0,005 \\
\hline & 20,0 & 40,0 & 0,03 & 0,5 & 60,0 & 35,0 \\
\hline$\hat{R}$ & 1,000 & 1,000 & 0,999 & 0,999 & 0,999 & 1,000 \\
\hline Média & 19,9 & 39,9 & 0,03 & 0,51 & 59,9 & 35,0 \\
\hline Moda & 19,9 & 40,0 & 0,03 & 0,51 & 59,9 & 35,0 \\
\hline HPD 95\% & {$[19,1 ; 20,8]$} & {$[39,0 ; 40,9]$} & {$[0,029 ; 0,031]$} & {$[0,45 ; 0,56]$} & {$[59,4 ; 60,4]$} & {$[34,6 ; 35,5]$} \\
\hline EMC & 0,01 & 0,01 & 0,00001 & 0,0003 & 0,003 & 0,005 \\
\hline \multirow[b]{2}{*}{$\hat{R}$} & 20,0 & 40,0 & 0,04 & 0,5 & 60,0 & 37,8 \\
\hline & 0,999 & 0,999 & 0,999 & 0,999 & 0,999 & 0,999 \\
\hline Média & 19,7 & 40,1 & 0,04 & 0,51 & 59,9 & 37,8 \\
\hline Moda & 19,7 & 40,1 & 0,04 & 0,51 & 59,9 & 37,8 \\
\hline HPD 95\% & {$[18,7 ; 20,8]$} & {$[39,1 ; 41,3]$} & {$[0,039 ; 0,042]$} & {$[0,45 ; 0,56]$} & {$[59,5 ; 60,3]$} & {$[37,3 ; 38,2]$} \\
\hline EMC & 0,001 & 0,001 & 0,00001 & 0,0001 & 0,003 & 0,004 \\
\hline \multirow{2}{*}{$\hat{R}$} & 20,0 & 60,0 & 0,03 & 0,5 & 80,0 & 42,5 \\
\hline & 1,003 & 1,004 & 1,001 & 1,000 & 1,000 & 1,000 \\
\hline Média & 19,9 & 60,0 & 0,03 & 0,51 & 79,8 & 42,5 \\
\hline Moda & 19,9 & 60,0 & 0,03 & 0,50 & 79,8 & 42,5 \\
\hline HPD 95\% & {$[18,9 ; 20,7]$} & {$[59,0 ; 61,0]$} & {$[0,029 ; 0,031]$} & {$[0,45 ; 0,56]$} & {$[79,3 ; 80,4]$} & {$[42,0 ; 42,9]$} \\
\hline EMC & 0,001 & 0,001 & 0,00001 & 0,0005 & 0,005 & 0,005 \\
\hline \multirow[b]{2}{*}{$\hat{R}$} & 20,0 & 60,0 & 0,04 & 0,5 & 80,0 & 46,7 \\
\hline & 1,004 & 1,003 & 1,003 & 1,000 & 1,001 & 1,002 \\
\hline Média & 19,7 & 60,2 & 0,04 & 0,51 & 79,8 & 46,7 \\
\hline Moda & 19,7 & 60,2 & 0,04 & 0,50 & 79,8 & 46,7 \\
\hline HPD 95\% & {$[18,5 ; 20,8]$} & {$[59,1 ; 61,3]$} & {$[0,039 ; 0,042]$} & {$[0,45 ; 0,57]$} & {$[79,4 ; 80,3]$} & {$[46,2 ; 47,1]$} \\
\hline EMC & 0,02 & 0,01 & 0,00001 & 0,0005 & 0,004 & 0,005 \\
\hline
\end{tabular}

${ }^{1}$ Degradabilidade efetiva com taxa de passagem de $5 \% / \mathrm{h} ;{ }^{2}$ Degradabilidade potencial.

Ciência Rural, v.39, n.7, out, 2009. 
Tabela 2 - Critério de Gelman e Rubin $(\hat{R})$, estimativas da média e moda a posteriori, intervalo de máxima densidade a posteriori (HPD) e Erro de Monte Carlo (EMC) para os dados simulados segundo o modelo de MCDONALD (1981).

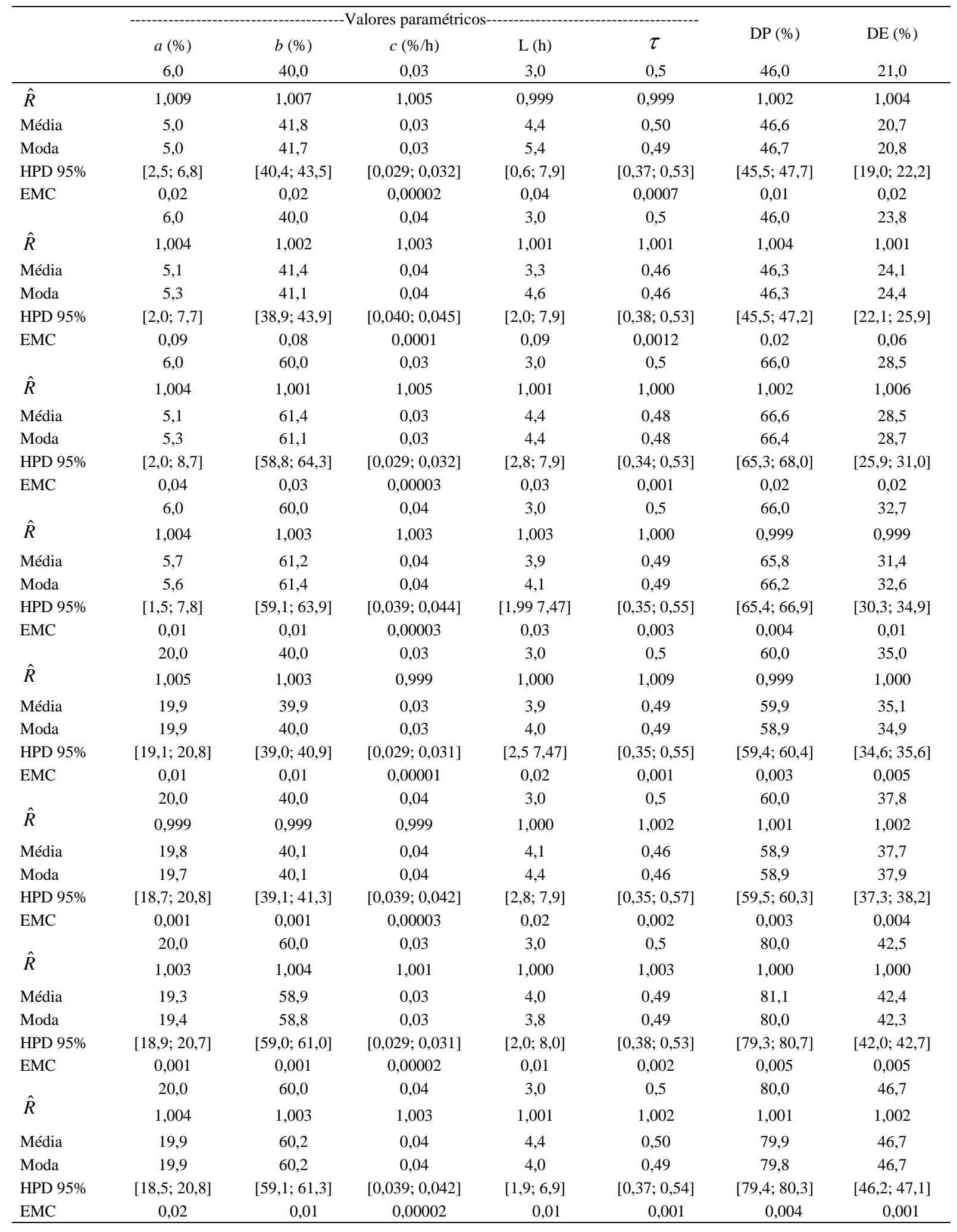

${ }^{1}$ Limites inferior e superior do intervalo HDP; ${ }^{2}$ Degradabilidade efetiva para taxa de passagem de $5 \% / \mathrm{h} ;{ }^{3}$ Degradabilidade potencial.

Ciência Rural, v.39, n.7, out, 2009. 
Tabela 3 - Valores estimados da média e moda a posteriori, intervalo de máxima densidade a posteriori (HPD), Erro de Monte Carlo (EMC) e valores para os critérios de convergência de Geweke e Raftery e Lewis no ajuste dos modelos de ORSKOV \& MCDONALD (1979) e MCDONALD (1981) aos dados reais.

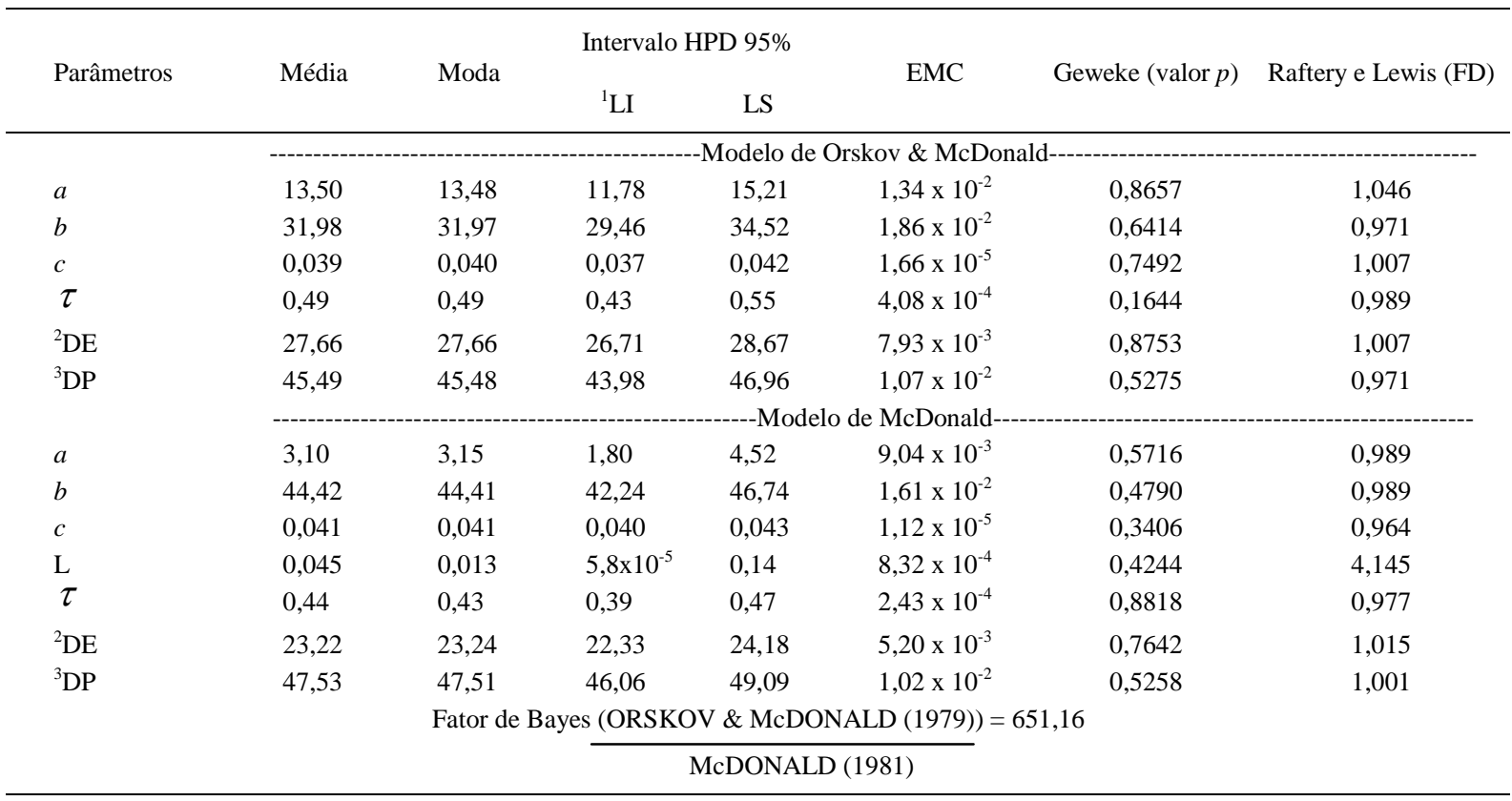

${ }^{1}$ Limites inferior e superior do intervalo HDP; ${ }^{2}$ Degradabilidade efetiva com taxa de passagem de $5 \% / \mathrm{h}$; ${ }^{3}$ Degradabilidade potencial.

das cadeias. Pela regra de decisão do critério de RAFTERY \& LEWIS (1992), na análise dos dados reais, verifica-se que as cadeias amostradas também atingiram a convergência (Tabela 3), ou seja, apresentaram o fator de dependência (FD) menor que cinco. Os valores encontrados para o Erro de Monte Carlo (EMC), tanto para os estudos de simulação, como para o ajuste dos modelos aos dados reais (Tabelas 1, 2 e 3), foram de baixa magnitude em relação à estimativa $a$ posteriori dos parâmetros, o que, segundo BLASCO et al. (2003), também assegura a convergência.

Uma vantagem da inferência bayesiana é que quaisquer combinações, lineares ou não, dos valores amostrais, uma vez calculada em cada passo de amostragem, geram diretamente um ponto amostral para a quantidade de interesse, com base na qual a inferência é bastante simples (ROSA, 1998). Assim, foram geradas cadeias para as degradabilidades efetiva (DE) e potencial (DP), as quais também tiveram suas convergências constatadas.

As estimativas da média e moda a posteriori, para o ajuste do modelo de Orskov e McDonald aos dados reais (Tabela 3), apresentam-se bastante próximas entre si, evidenciando distribuições do tipo simétricas, para todos os parâmetros. Nesses casos, um resumo pontual da informação contida nas distribuições a posteriori pode ser dado, tanto pela média como pela moda a posteriori. No ajuste do modelo de McDonald aos dados reais (Tabela 3), observa-se que apenas para o parâmetro $L$ a distribuição apresenta-se assimétrica à direita. Em estudos futuros, podem ser consideradas, para os parâmetros com distribuições assimétricas, distribuições a priori do tipo Gama.

As estimativas obtidas para os parâmetros dos modelos de Orskov e McDonald e McDonald, por meio da abordagem bayesiana, mostraram-se bastante coerentes com os valores relatados na literatura. $O$ valor encontrado para a fração potencialmente degradável (b) da FDN (Tabela 3) foi de, aproximadamente, 32\%. No entanto, a degradabilidade efetiva (DE) foi próxima de $28 \%$, mostrando que o alimento pode permanecer mais tempo no rúmen para atingir seu máximo potencial de degradação.

ASSIS et al. (1999), avaliando a degradabilidade efetiva da FDN do capim Tifton85 cortado aos 35 dias, encontraram, para esse parâmetro, um valor de 34,26\%. MENDONÇA (1983) afirma que a composição química das forrageiras é afetada, principalmente, pela idade da planta e pode variar entre espécies e cultivares. $\mathrm{O}$ avanço da idade interfere no valor nutritivo de gramíneas forrageiras por meio de 
mudanças na composição química, devido ao aumento do teor de fibra e ao decréscimo do teor de proteína bruta.

Para a fração solúvel (parâmetro $a$ ), foi encontrado, neste estudo, valor de 14\% para a gramínea coastcross cortada aos 120 dias. SOARES et al. (2008), utilizando capim-elefante fornecido picado para vacas mestiças lactantes e canuladas no rúmen, encontraram, para fração solúvel (parâmetro $a$ ), um valor próximo a 19\% para o capim-elefante, porém cortado aos 60 dias. SAVIAN et al. (2007a), estudando a degradabilidade do capim-coastcross, encontraram para a fração solúvel de FDN o valor de 14,2\%.

Com relação à taxa de degradação da FDN, os resultados mostram, para esse parâmetro, estimativas em torno de $4,0 \% / \mathrm{h}$, as quais estão dentro da faixa sugerida pelo CNCPS, entre 3,0\% e 8,1\% $\mathrm{h}^{-1}$, para plantas tropicais.

O resultado da comparação dos modelos, para os dados reais (Tabela 3), por meio do fator de Bayes, indica que o modelo de Orskov e McDonald é mais plausível que o modelo de McDonald. Segundo a interpretação sugerida por JEFFREYS (1961), a magnitude atingida pelo fator de Bayes demonstra que e a evidência a favor do modelo de Orskov e McDonald é considerada muito forte, uma vez que o fator resultou em um valor superior a 100.

\section{CONCLUSÕES}

A metodologia bayesiana mostrou-se eficiente no estudo de modelos de degradabilidade ruminal, sendo avaliada e comprovada pelo estudo de simulação, apresentando estimativas bem próximas ao valor verdadeiro no ajuste de ambos os modelos.

O modelo de Orskov e McDonald, na descrição dos dados de porcentagem de degradação da fibra em detergente neutro do capim-coastcross, com idade de corte de 120 dias, foi mais plausível que o modelo de McDonald.

\section{AGRADECIMENTO}

À Fundação de Amparo à Pesquisa do Estado de Minas Gerais (FAPEMIG), pelo suporte financeiro concedido para a realização deste trabalho.

\section{REFERÊNCIAS}

ASSIS, M.A. et al. Degradabilidade in situ de gramíneas do gênero Cynodon submetidas ou não a adubação nitrogenada. Acta Scientiarum, Maringá, v.21, n.3, p.657-663, 1999.

BLASCO, A. et al. Bayesian analysis of the effect of selection for growth rate curves in rabbits. Genetics Selection Evolution, Les Ulis, v.35, n.1, p.21-41, 2003.
GELMAN, A.; RUBIN, D. B. Inference from iterative simulation using multiple sequences. Statistical Science, Hayward, v.7, n.4, p.457-511, 1992.

GEWEKE, J. Evaluating the accuracy of sampling-based approaches to the calculation of posterior moments. In: BERNARDO, J.M. et al. Bayesian statistics. New York: Oxford University, 1992. Cap.4, p.625-631.

GUEDES, M.H.P. et al. Análise Bayesiana da curva de crescimento de cordeiros da raça Santa Inês. Arquivo Brasileiro de Medicina Veterinária e Zootecnia, Belo Horizonte, v. 57, n. 3, p. 415-417. 2005. Disponível em <http://www.scielo.br/ s ci el o.php? s c ri p t = s ci_art text \& pid=S 0102 09352005000300024\&lng=pt\&nrm=iso $>$. Acesso em: 12 maio 2009. doi: 10.1590/S0102-09352005000300024.

JEFFREYS, H. Theory of probability. Oxford: Claredon, 1961. 325p.

KASS, R.E.; RAFTERY, A.E. Bayes factor. Journal of the American Statistical Association, Alexandria, v.90, n.430, p.773-795, 1995.

MARTINS FILHO, et al. Abordagem Bayesiana das curvas de crescimento de duas cultivares de feijoeiro. Ciência Rural, Santa Maria, v. 38, n. 6, p. 1516-1521, 2008. Disponível em $<$ http:/www.scielo.br/scielo.php?script=sci_arttext\&pid=S010384782008000600004\&lng=pt\&nrm=iso $>$. Acesso em: 12 maio 2009. doi: 10.1590/S0103-84782008000600004.

McDONALD, I. A revised model for the estimation of protein degradability in the rumen. Journal of Agricultural Science, Cambridge, v.96, n.1, p.251-252, 1981.

MENDONÇA, J.F.B. Rendimento e valor nutritivo do campim elefante (Pennisetum purpureum Schum) cv. Cameroon. 1983. 110f. Dissertação (Mestrado em Zootecnia) - Escola Superior de Agricultura de Lavras.

MERTENS, D.R. Rate and extent of digestion. In: FORBES, J.M.; FRANCE, J. Qualitative aspects of ruminant digestion and metabolism. Wallingford, UK: Cambridge University, 1993. Cap.2, p.13-51.

MUNIZ, J.A. et al. Evaluation of lactation curve of lowyelding gir cows: a Bayesian approach. Acta Scientiarum Animal Sciences, Maringá, v.29, n.1, p.79-83, 2007.

NOGUEIRA, D. A. et al. Avaliação de critérios de convergência para o método de Monte Carlo via Cadeias de Markov. Revista Brasileira de Estatística, Rio de Janeiro, v.65, n.24, p.5988, 2004.

ORSKOV, E.R. Protein nutrition in ruminants. London: Academic, 1982. 160p.

ORSKOV, E.R.; McDONALD, I. The estimation of protein degradability in the rumen from incubation measurements weighted according to rate of passage. Journal of Agricultural Science, Cambridge, v.92, n.2, p.499-503, 1979.

PAULINO, C. et al. Estatística Bayesiana. Lisboa: Fundação Calouste Gulbenkian, 2003. 446p.

R Development Core Team. R: a language and environment for statistical computing. Vienna, Austria: R Foundation for 
Statistical Computing. Disponível em: <http://www.Rproject.org>. Acesso em: 12 mar. 2007.

RAFTERY, A.L.; LEWIS, S. Comment: one long run with diagnostics: implementation strategies for Markov chain Monte Carlo. Statistical Science, Hayward, v.7, n.4, p.493-497, 1992.

REIS, S.T. dos. Valor nutricional de gramíneas tropicais em diferentes idades de corte. 2000. 99f. Dissertação (Mestrado em Zootecnia) - Curso de Pós-graduação em Zootecnia, Universidade Federal de Lavras, Lavras, MG.

ROSA, G.J.M. Análise Bayesiana de modelos lineares mistos robustos via Amostrador de Gibbs. 1998. $57 \mathrm{f}$ Tese (Doutorado em Estatística e Experimentação Agronômica) - Curso de Pós-graduação em Estatística e Experimentação Agronômica, Universidade de São Paulo, Piracicaba, SP.

SAVIAN, T.V. et al . Degradability study of neutral detergent fiber of coast cross (Cynodon dactylon $x$ Cynodon nlemfunensis). Ciência e Agrotecnologia, Lavras, v. 31, n. 4, p. 1184-1190. 2007a. Disponível em <http://www.scielo.br/ scielo.ph p ? script=sci_art text\&pid = S 1413 $70542007000400035 \& \operatorname{lng}=p t \& n r m=i s o>$. Acesso em: 12 maio 2009. doi: 10.1590/S1413-70542007000400035.

SAVIAN, T.V. et al. Comparison of two mathematical models in the description of in situ degradability. Revista Brasileira de Ciência Veterinária, v.14, n.2, p.72-78, 2007b.
SAVIAN, T.V.; MUNIZ, J.A. A study of in situ degradability: heterogeneity of variances and correlated errors. Scientia Agricola, Piracicaba, v. 64, n. 5, p. 548-554, 2007c. Disponível em <http://www.scielo.br/scielo.php?script=sci_arttext\&pid=S010390162007000500013\&lng=pt\&nrm=iso>. Acesso em: 12 maio 2009. doi: 10.1590/S0103-90162007000500013.

SILVA, F.F. et al. Abordagem Bayesiana da curva de lactação de cabras Saanen de primeira e segunda ordem de parto. Pesquisa Agropecuária Brasileira, Brasília, v. 40, n. 1, p. 27-33, 2005. Disponível em: $<$ http://www.scielo.br/scielo.php?script=sci_arttext\&pid=S0100 204X2005000100004\&lng=pt\&nrm=iso>. Acesso em: 12 maio 2009. doi: 10.1590/S0100-204X2005000100004.

SILVA, F.F. et al. Comparação bayesiana de modelos de previsão de diferenças esperadas nas progênies no melhoramento genético de gado Nelore. Pesquisa Agropecuária Brasileira, Brasília, v.43, n.1, p. 37-45, 2008. Disponível em <http:// www. scielo.br/scielo.php?script $=$ sci_arttext \&pid $=$ S0100204X2008000100006\&lng=pt\&nrm=iso $>$. Acesso em: 12 maio 2009. doi: 10.1590/S0100-204X2008000100006.

SILVA, N.A. et al. Estudo de parâmetros de crescimento de bezerros Nelore por meio de um modelo de regressão linear: uma abordagem bayesiana. Ciência Animal Brasileira, Goiânia, v.7, n.1, p.57-65, 2006.

SOARES, J.P.G.; BERCHIELLI, T.T. et al. Comparações entre equações de predição de consumo de capim-elefante cortado para vacas lactantes. Archivos de Zootecnia, Córdoba, v.57, p.235-246, 2008 